\title{
Quantum Algorithmic Readout in Multi-Ion Clocks
}

\author{
M. Schulte, ${ }^{1}$ N. Lörch, ${ }^{1,4}$ I. D. Leroux, ${ }^{2}$ P. O. Schmidt, ${ }^{2,3}$ and K. Hammerer ${ }^{1}$ \\ ${ }^{1}$ Institute for Theoretical Physics and Institute for Gravitational Physics (Albert-Einstein-Institute), \\ Leibniz University Hannover, Callinstrasse 38, 30167 Hannover, Germany \\ ${ }^{2}$ QUEST Institut, Physikalisch-Technische Bundesanstalt, 38116 Braunschweig, Germany \\ ${ }^{3}$ Institute for Quantum Optics, Leibniz. University Hannover, Welfengarten 1, 30167 Hannover, Germany \\ ${ }^{4}$ Department of Physics, University of Basel, Klingelbergstrasse 82, 4056 Basel, Switzerland
}

(Received 28 January 2015; published 8 January 2016)

\begin{abstract}
Optical clocks based on ensembles of trapped ions promise record frequency accuracy with good short-term stability. Most suitable ion species lack closed transitions, so the clock signal must be read out indirectly by transferring the quantum state of the clock ions to cotrapped logic ions of a different species. Existing methods of quantum logic readout require a linear overhead in either time or the number of logic ions. Here we describe a quantum algorithmic readout whose overhead scales logarithmically with the number of clock ions in both of these respects. The scheme allows a quantum nondemolition readout of the number of excited clock ions using a single multispecies gate operation which can also be used in other areas of ion trap technology such as quantum information processing, quantum simulations, metrology, and precision spectroscopy.
\end{abstract}

DOI: 10.1103/PhysRevLett.116.013002

Tremendous progress has recently been made in optical frequency metrology [1,2]. Optical clocks now reach fractional frequency inaccuracies and instabilities in the $10^{-18}$ regime [3-8], outperforming Cs fountain clocks by 2 orders of magnitude and vying to serve as a new definition of the SI second $[9,10]$. Among the promising candidates for such a redefinition are ion-based frequency standards featuring very small systematic frequency shifts. However, the poor signal-to-noise ratio of single-ion systems entails averaging times of many weeks to reach a fractional uncertainty of $10^{-18}[11,12]$. Clocks based on strings of ions confined in a linear trap promise to overcome this limitation [13,14]. Because of unavoidable electric field gradients in such a trap, suitable clock ion species may have only negligible electric quadrupole moments to avoid systematic frequency shifts [15]. This requirement is met by group 13 ion species [16], as well as some highly charged ions [17-20]. Most of these candidates lack a suitable transition for laser cooling and state detection, so that quantum logic spectroscopy [21] is required for readout. In quantum logic spectroscopy the internal state of the clock ion is transferred by a series of laser pulses onto a logic ion of a different species, whose internal state can be efficiently detected. However, existing methods for quantum logic readout require a large overhead in either time or logic ions when an ensemble of clock ions is used.

Here, we suggest using a quantum algorithmic readout, cf. Fig. 1, which maps the clock signal (that is, the number of clock ions left in the excited state by the spectroscopy pulses) on a string of logic ions in binary representation and therefore requires a number of logic ions scaling logarithmically with the number of clock ions. Our approach is based on an algorithm originally introduced in the context of entanglement concentration protocols [22,23], which can be implemented efficiently with a number of elementary gate operations scaling likewise logarithmically with the number of clock ions. Moreover, we show that the algorithm can be executed by means of the multi-ion Mølmer-Sørensen (MS) gate [24-27], a well-established tool for quantum control of ion crystals $[28,29]$. In particular, we demonstrate that only a single application of a MS gate involving both logic and clock ions is necessary. Such multispecies multi-ion gate operations are under intense experimental investigation at the moment [30], and constitute the most costly resource in the current

(a)

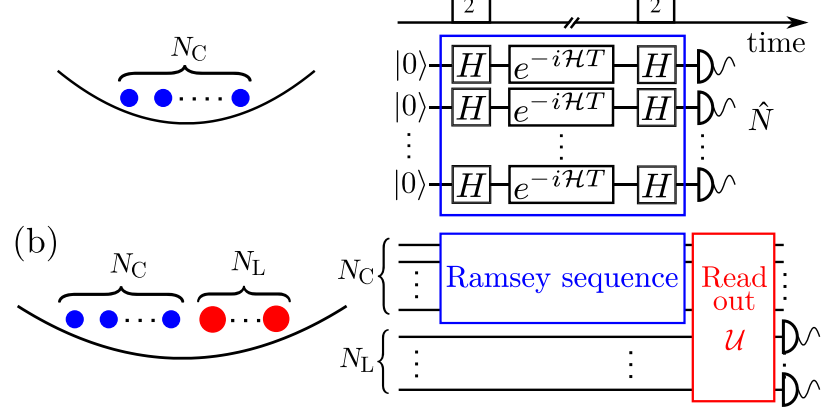

FIG. 1. (a) In an atomic clock based on $N_{C}$ trapped ions the frequency of a laser is locked to an atomic transition, e.g., in a Ramsey sequence of two $\pi / 2$ pulses (Hadamard gates $H$ ) enclosing a free evolution time $T$. Measurement of the number $\hat{N}$ of clock ions in $|1\rangle$ yields an error signal for the deviation of the laser from resonance. (b) For ion species lacking the cycling transition needed for direct state detection, a quantum algorithmic readout can be used to map $\hat{N}$ onto $N_{L}$ cotrapped logic ions of a different species whose state can be detected efficiently. 
context due to the complexity of the normal mode structure in multispecies ion crystals. We discuss the feasibility of our approach in a case study of $3 \mathrm{Al}^{+}$and $2 \mathrm{Ca}^{+}$ions taking into account the full spectrum of motional normal modes.

The suggested readout method actually realizes a quantum nondemolition (QND) measurement on the clock ions, and therefore opens up the possibility to devise more sophisticated clock protocols where subsets of clock ions are interrogated repeatedly. Beyond frequency metrology the QND character of our readout technique makes it directly applicable to syndrome measurements and error correction schemes for quantum computations and simulations with single- or multispecies ion crystals [31]. In a more general perspective, this work contributes to the development of hybrid systems striving to combine the complementary strengths of different physical components for quantum information processing, quantum simulations, and quantum metrology [32,33].

Working principle of ion clocks with direct readout.We consider a string of $N_{C}$ clock ions with a narrow-band optical transition of frequency $\omega_{0}$ between two internal states $|0\rangle$ and $|1\rangle$ which provides the frequency reference for the clock. The goal is to stabilize to this transition frequency $\omega_{0}$ a laser field of frequency $\omega$ with an (unknown) frequency offset $\Delta=\omega-\omega_{0}$. To this end, pulses of light from the laser drive the clock ions, transferring them from $|0\rangle$ to the excited state $|1\rangle$ with a frequency-dependent probability. In the simplest schemes, such as Rabi and Ramsey interrogation, this probability is independent for each ion. The clock readout then consists of measuring the number of excited ions $\hat{N}=\sum_{i=1}^{N_{C}}|1\rangle_{i}\langle 1|$ and using it to infer the excitation probability and hence the detuning $\Delta$, which can then be corrected [34]; cf. Fig. 1(a). As explained above, a direct measurement of the excited state population $\hat{N}$ is impractical for many interesting species of clock ion. Instead, one can map $\hat{N}$ onto an ensemble of $N_{L}$ cotrapped logic ions which can be detected efficiently, as illustrated in Fig. 1(b). In direct extension of established readout techniques based on quantum logic $[21,36]$, one could use as many logic ions as clock ions $\left(N_{L}=N_{C}\right)$, performing successive quantum gate operations to transfer the state of each clock ion to the corresponding logic ion. Alternatively, one could use a single logic ion, transferring and measuring the state of each clock ion in turn. This imposes a long readout time as the ion crystal must be cooled between each measurement and subsequent state swap operation. Both strategies have prohibitive overhead in additional ions or number of gate operations and readout time. The last is crucial because time spent on readout adds to the clock cycle's dead time and, thus, through the Dick effect, to the clock instability [37,38].

Quantum algorithmic readout.-From the perspective of quantum information theory, the quantity of interest- the number of clock ions in state $|1\rangle$-is the Hamming weight of the string of $N_{C}$ quantum bits (a number with $N_{C}+1$ possible values between 0 and $N_{C}$ ). In the context of entanglement concentration protocols, a quantum algorithm has been developed for the indirect determination of the Hamming weight of a quantum bit string [22,23]. The algorithm uses an ancillary string of $N_{L}=$ $\left\lceil\log _{2}\left(N_{C}+1\right)\right\rceil$ quantum bits on which the Hamming weight of the $N_{C}$ primary quantum bits is stored in binary representation. Thus, the necessary number $N_{L}$ of logic ions (ancillary quantum bits) scales logarithmically with the number of clock ions. Suitable combinations of clock and logic ion numbers $\left(N_{C}, N_{L}\right)$ are, for example, $(3,2)$, $(7,3)$, and $(15,4)$. The algorithmic readout is shown in a circuit diagram for the simple case $\left(N_{C}, N_{L}\right)=(3,2)$ in Fig. 2(a), and for the general case in Ref. [39]. Given $N_{C}$ quantum bits (clock ions) in any state $|N\rangle_{C}$ with Hamming weight $N$ and $N_{L}$ ancillary bits (logic ions) initialized in $|00 \ldots 0\rangle_{L}$, the algorithm effects a unitary transformation $\mathcal{U}$ such that $\mathcal{U}|N\rangle_{C}|00 \ldots 0\rangle_{L}=|N\rangle_{C}\left|i_{1} i_{2} \ldots i_{N_{L}}\right\rangle_{L}$, where the bit string $i_{1} i_{2} \ldots i_{N_{L}}\left(i_{n}=0,1\right)$ gives the binary representation of the Hamming weight of the state of clock ions, $N=\sum_{n=1}^{N_{L}} 2^{n-1} i_{n}$. We will denote by $|\underline{N}\rangle_{L}=\left|i_{1} i_{2} \ldots i_{N_{L}}\right\rangle_{L}$ the state of logic ions representing $N$. The state of the clock ions after one clock cycle is a superposition $|\psi(\Delta)\rangle_{C}=$ $\sum_{N=0}^{N_{C}} c_{N}(\Delta)|N\rangle_{C}$, where the dependence of the amplitudes $c_{N}(\Delta)$ on the detuning carries the clock signal [47]. Application of the readout algorithm generates an entangled state of clock and logic ions:

$$
|\Psi(\Delta)\rangle=\mathcal{U}|\psi(\Delta)\rangle_{C}|\underline{0}\rangle_{L}=\sum_{N=0}^{N_{C}} c_{N}(\Delta)|N\rangle_{C}|\underline{N}\rangle_{L} .
$$

From a measurement in the $\{|0\rangle,|1\rangle\}$ basis of each logic ion one can extract the observable corresponding to the estimated Hamming weight of the clock ions, $\hat{N}_{\text {est }}=\sum_{n=1}^{N_{L}} 2^{n-1}|1\rangle_{n}\left\langle 1\left|=\sum_{N=0}^{N_{C}} N\right| \underline{N}\right\rangle_{L}\langle\underline{N}|$. In a perfect implementation the measurement of $\hat{N}_{\text {est }}$ on the logic ions exhibits exactly the same statistics as measuring $\hat{N}$ on the clock ions directly. In particular, the error signal can be extracted from $N(\Delta)=\left\langle\Psi(\Delta)\left|\hat{N}_{\text {est }}\right| \Psi(\Delta)\right\rangle$ and used to correct $\omega$ exactly as in a direct readout.

Implementation based on Mølmer-Sørensen gates.Following Refs. [22,23], the operation $\mathcal{U}$ can be implemented by a sequence of quantum Fourier transforms and controlled-phase gates as shown in Fig. 2(a). This requires a number of gates linear in $N_{C}$, and so provides little advantage over the complete state swap mentioned above (apart from the reduction in the number of logic ions). Fortunately, the algorithm can be decomposed much more efficiently using multi-ion Mølmer-Sørensen gates [24-26]. MS gates involve driving the ions simultaneously with bichromatic laser fields on red and blue sideband transitions to collective modes of vibrations for a duration $\tau$, and generate unitary transformations $U_{\mathrm{MS}}=\exp \left[-i\left(S_{C C}+S_{L L}+S_{C L}\right)\right]$ on the string of $N_{C}+N_{L}$ two-level systems, where $[39,48]$

$$
S_{\alpha \beta}=\sum_{i=1}^{N_{\alpha}} \sum_{j=1}^{N_{\beta}} \frac{\Gamma_{i j}^{\alpha \beta}}{\Delta_{i j}^{\alpha \beta}} \sigma_{\alpha i}^{x} \sigma_{\beta j}^{x} .
$$


(a) Ramsey sequence

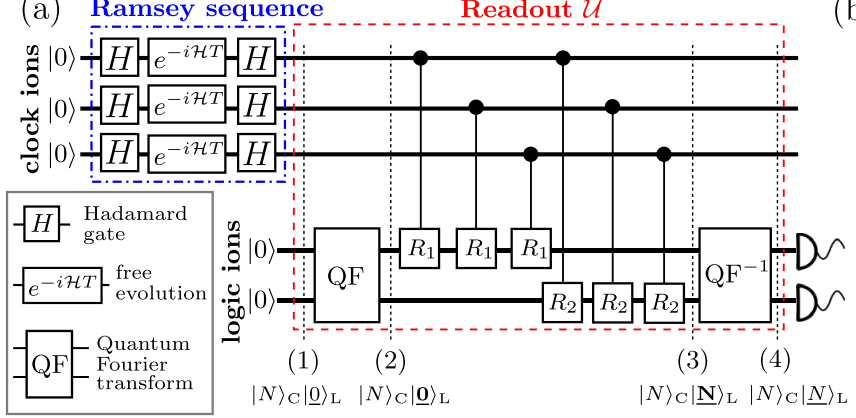

(b)



(1) (2)

(3)

FIG. 2. Quantum algorithmic readout illustrated for $N_{C}=3$ clock and $N_{L}=2$ logic ions: after a Ramsey sequency (blue, dash-dotted box) the clock ions are in a superposition of states $|N\rangle_{C}$, where $N \in\left[0, N_{C}\right]$ is the number of ions in excited states $|1\rangle$ (their Hamming weight). A quantum algorithmic readout $\mathcal{U}$ (red, dashed box) maps $N$ in binary representation onto a number $N_{L}=\left\lceil\log _{2}\left(N_{C}+1\right)\right\rceil$ of logic ions. Detection of the logic ions in the $\{|0\rangle,|1\rangle\}$ basis provides the number $N$. (a) Quantum algorithm for the indirect measurement of the Hamming weight, taken from Refs. [22,23]. (1) Logic ions are initialized in $|\underline{0}\rangle_{L}=|00\rangle_{L}$ (states $|\underline{N}\rangle_{L}$ denote the binary representation of $N$ ). (2) The state is quantum Fourier transformed into $|\underline{\mathbf{0}}\rangle_{L}$. (3) Controlled-phase gates rotate the state in Fourier space to $|\underline{\mathbf{N}}\rangle_{L}$ for a state $|N\rangle_{C}$ of clock ions. (4) An inverse Fourier transform yields the state $|\underline{N}\rangle_{L}$ of logic ions. (b) The same algorithm decomposed in terms of multi-ion Mølmer-Sørensen (MS) gates. The Hadamard gates shown in gray need not be executed. Removing Hadamard gates displayed with dashed boxes merges the algorithmic readout with the Ramsey sequence, and requires the laser fields in the MS gates to be phase coherent with the Hadamard gates in the Ramsey sequence. Explicit, general forms of all gates are given in Ref. [39].

Here, $\sigma_{\alpha i}^{x}$ denotes the Pauli $x$ operator for ion $(\alpha, i)$, where $\alpha=C, L$ and $i=1, \ldots, N_{\alpha}$, and the frequencies $\Gamma_{i j}^{\alpha \beta}$ and $\Delta_{i j}^{\alpha \beta}$ are defined through

$\Gamma_{i j}^{\alpha \beta}=\int_{0}^{\tau} d t \Omega_{\alpha i}(t) \Omega_{\beta j}(t), \quad \frac{1}{\Delta_{i j}^{\alpha \beta}}=\sum_{k=1}^{N_{L}+N_{C}} \frac{\eta_{\alpha i}^{k} \eta_{\beta j}^{k}}{\delta_{k}}$.

We assume that the Rabi frequencies $\Omega_{\alpha i}(t)$ can be adjusted separately for each ion in transverse illumination, and may also be tuned in time. In the definition of $\Delta_{i j}^{\alpha \beta}$ in Eq. (2) the $\eta_{\alpha i}^{k}$ are Lamb-Dicke factors and $\delta_{k}$ detunings with respect to the $k$ th transverse normal mode of (angular) frequency $\nu_{k}$; cf. Fig. 3(a). The detunings have to satisfy $\delta_{k} \gg\left|\Omega_{\alpha i} \eta_{\alpha i}^{k}\right|$ and we suppose $\delta_{1}>0$. A decomposition of the desired transformation $\mathcal{U}$ in terms of multi-ion MS gates and singleion rotations is shown in Fig. 2(b). Remarkably, only a single two-species MS gate is required when the interaction between clock and logic ions, described by $S_{C L}$ in Eq. (1), is tuned such that

$$
\Gamma_{i j}^{C L}=\pi 2^{-(j+2)} \Delta_{i j}^{C L},
$$

where $i=1, \ldots, N_{C}, j=1, \ldots, N_{L}$ [49]. Equation (3) ensures that the MS gate executes the controlled-phase gates between logic and clock ions as shown in Fig. 2(a). Read as a matrix equation, it expresses $N_{C} \times N_{L}$ constraints where the right-hand side is fixed for a given configuration of the ion string and carrier detuning $\delta=\delta_{k}+\nu_{k}$, while the left-hand side has to be adapted accordingly through the correct choice of Rabi frequencies $\Omega_{\alpha i}(t)$ and the gate duration $\tau$. In addition to the interactions between clock and logic ions $\left(S_{C L}\right)$, the gate also induces interactions among clock ions $\left(S_{C C}\right)$ and logic ions $\left(S_{L L}\right)$ only. The interactions on the clock ions have no influence on the readout results and can therefore be neglected. On the other hand, interactions between logic ions have to be compensated using $N_{L}-1$ gates that involve only logic ions [39]. The inverse Fourier transform on the string of logic ions involves $N_{L}-1$ single-species MS gates giving a total number $2 N_{L}-1$ of multiqubit gates [39] that grows logarithmically with the number of clock ions $N_{C}$.

The remaining challenge is to find experimental parameters that satisfy Eq. (3). If each ion can be addressed individually, the time dependence of $\Omega_{\alpha i}(t)$ can be exploited to exactly fulfill Eq. (3); see Ref. [39] for details. Here, we will instead focus on finding an approximate solution to condition (3) using only time-independent Rabi frequencies. This restriction substantially simplifies the experimental implementation, which can then employ a single laser beam for each species with a tailored intensity profile, rather than
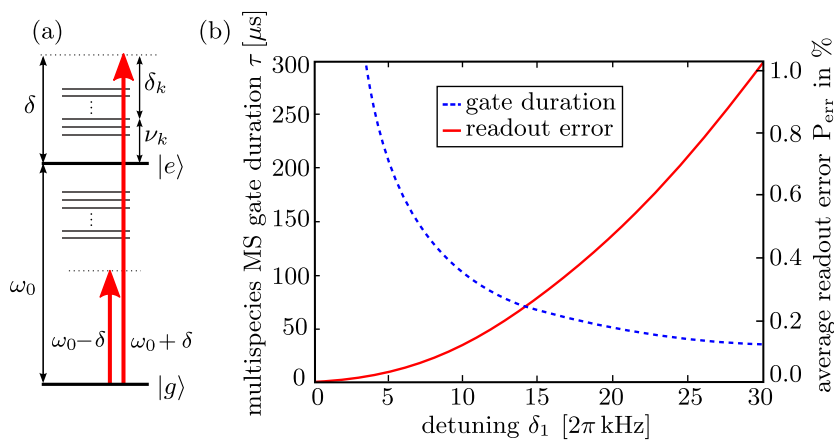

FIG. 3. (a) Schematic level diagram with relevant frequencies during MS gates: atomic transition $\omega_{0}$, bichromatic driving fields at $\omega_{0} \pm \delta$, detuning $\delta_{k}$ from sideband $\nu_{k}$ due to collective mode $k$. (b) Average readout error probability $P_{\text {err }}$ (red, solid line) and multispecies MS gate duration $\tau$ (blue, dashed line) versus detuning $\delta_{1}$. 
requiring full individual addressing with independent pulse shaping for each ion. In this case the left-hand side of Eq. (3) becomes $\Gamma_{i j}^{C L}=\Omega_{C i} \Omega_{L j} \tau$, which is patently a rank-one matrix while the rhs of Eq. (3) generically has full rank $\left(N_{L}\right)$. However, if the detuning $\delta_{1}$ is small on the scale of the mode spacing $\Delta \nu=\nu_{1}-\nu_{2}$, the ion interactions in the MS gate are mostly mediated via the highest-frequency collective mode. In this regime the rhs of Eq (3) will be almost of rank-one in the sense that its singular value decomposition exhibits one dominating singular value. Accordingly, the rhs of Eq. (3) can be well approximated in terms of a rank-one matrix by determining its singular value decomposition and setting all but the largest singular value to zero as detailed in Ref. [39]. We emphasize that this is not equivalent to simply dropping lower-lying modes [which would correspond to restricting the summation in Eq. (2) to $k=1$ ].

Case study with $\mathrm{Al}^{+}$and $\mathrm{Ca}^{+}$ions. - In the remainder of this Letter we discuss in more detail the realization of multispecies MS gates with constant Rabi frequencies for the concrete case of three $\mathrm{Al}^{+}$clock ions and two $\mathrm{Ca}^{+}$logic ions. We assume the ions are held in a linear rf Paul trap with soft confinement along the crystal axis ( $z$ axis) and much tighter confinements in the transverse directions that effectively restrict oscillations to only one direction ( $x$ axis). An important requirement for the MS gates is truly collective normal modes; i.e., each ion must be involved in the motion to interact with the others. For the case of multispecies crystals, these modes are found in a stable regime close to the critical confinement where the linear chain changes to a zigzag configuration [31,50,51]. In Ref. [39] we show for the case of $\mathrm{Al}^{+}-\mathrm{Ca}^{+}$crystals that for moderate trap asymmetry the highest-frequency mode is sufficiently collective and exhibits Lamb-Dicke factors on the order of $10^{-1}$ for clock ions and $10^{-3}-10^{-2}$ for logic ions while exhibiting a substantial frequency gap $\Delta \nu \simeq 2 \pi 500 \mathrm{kHz}$ to the next mode. The approximate solutions to Eq. (3) will be applicable when the detuning in the multispecies MS gate is small enough, that is, $\delta_{1} \ll \Delta \nu$. For a given detuning $\delta_{1}$, we require a gate duration $\tau=2 \pi / \delta_{1}$ in order to suppress spurious effects of off-resonant terms during the MS gate, which implies a time scale in the sub-ms regime for the gate.

In order to assess the performance of our readout strategy, we evaluate the average error probability defined as $P_{\text {err }}=1-\sum_{N=0}^{N_{C}} p_{N} P\left[N \mid \rho_{C}(N)\right]$. Here, $P\left[N \mid \rho_{C}(N)\right]$ is the conditional probability to correctly receive the Hamming weight $N$ as a result of the quantum algorithmic readout given that exactly $N$ ions were in the excited state in the initial state of clock ions $\rho_{C}(N)$. The $p_{N}$ denote the a priori probabilities for the states $\rho_{C}(N)$. In the context of ion clocks, the maximum error signal will be attained when each clock ion is in an equal superposition of $|0\rangle$ and $|1\rangle$, which implies that $p_{N}=\left(\begin{array}{c}N_{C} \\ N\end{array}\right) / 2^{N_{C}}$ and $\rho_{C}(N)$ is the symmetric Dicke state with $N$ clock ions in $|1\rangle$. The conditional probabilities are evaluated as $P\left[N \mid \rho_{C}(N)\right]=$ $\operatorname{tr}_{C L}\left\{|\underline{N}\rangle_{L}\left\langle\underline{N}\left|\mathcal{U} \rho_{C}(N) \otimes\right| 00\right\rangle_{L}\langle 00| \mathcal{U}^{\dagger}\right\}$, where $\mathcal{U}$ is the circuit shown in Fig. 2(b). For a given detuning $\delta_{1}$ the Rabi frequencies in the single multispecies MS gate follow from the vectors corresponding to the largest singular value of the rhs of Eq. (3), as explained above. Figure 3 shows the overall error probability $P_{\text {err }}$ and the gate duration $\tau$ versus detuning $\delta_{1}$ in the MS gate with otherwise ideal processes. For example, a detuning $\delta_{1}=2 \pi 20 \mathrm{kHz}$ corresponds to a gate duration of $\tau=50 \mu \mathrm{s}$ and an error probability of $0.5 \%$, which is achieved with Rabi frequencies $\left(\Omega_{L 1}, \Omega_{L 2}\right)=$ $2 \pi(730.34,363.81) \mathrm{kHz}$ and $\left(\Omega_{C 1}, \Omega_{C 2}, \Omega_{C 3}\right)=2 \pi(49.71$, $45.84,53.81) \mathrm{kHz}$ for logic and clock ions, respectively. In this example, the carrier transition is driven off resonantly with a Rabi frequency to a detuning ratio of at most $\Omega_{\alpha i} / \delta=0.23$. However, the associated ac Stark shifts cancel due to the bichromatic, red- and blue-detuned drive and can therefore be neglected. At the same time, the $50 \mu \mathrm{s}$ gate duration is short enough compared to the $1.17 \mathrm{~s} D_{5 / 2}$ state lifetime in $\mathrm{Ca}^{+}$that readout errors due to spontaneous emission will be on the per mill level. The Rabi frequencies required in the present example can be tailored by implementing the gate with a tightly focused $\mathrm{TEM}_{10}$ laser beam addressing the three clock ions, such that each ion is located at a position in the transverse intensity profile corresponding to the Rabi frequency given above.

The more complex spatial structure of Rabi frequencies needed in a longer string of ions can be engineered with spatial light modulators [52] or multichannel acousto-optical modulators. Combined with the freedom to permute clock and logic ions and to choose different solutions to Eq. (3), we expect experimentally feasible implementations for more than 15 clock and 4 logic ions. Importantly, readout errors will affect only the stability, not the accuracy, of the ion clock.

Conclusion.-The quantum algorithmic readout suggested here performs a QND measurement of the Hamming weight of clock ions and may therefore allow more complex clock protocols using repeated readouts of (sub)ensembles of ions or preparation of the clock ions in Dicke states for nonclassical frequency metrology. We envision that the techniques developed here in the context of atomic clocks may prove useful also in other areas of ion trap technology, such as quantum information processing, quantum simulations, metrology, and precision spectroscopy.

We acknowledge support from DFG through QUEST. I. D. L. acknowledges support from the Alexander von Humboldt Foundation. This work was supported by the European Metrology Research Programme (EMRP) in project SIB04. The EMRP is jointly funded by the EMRP participating countries within EURAMET and the European Union.

[1] N. Poli, C. W. Oates, P. Gill, and G. M. Tino, Riv. Nuovo Cimento 36, 555 (2013).

[2] A. D. Ludlow, M. M. Boyd, J. Ye, E. Peik, and P. O. Schmidt, Rev. Mod. Phys. 87, 637 (2015). 
[3] T. Rosenband, D. B. Hume, P. O. Schmidt, C. W. Chou, A. Brusch, L. Lorini, W. H. Oskay, R. E. Drullinger, T. M. Fortier, J. E. Stalnaker, S. A. Diddams, W. C. Swann, N. R. Newbury, W. M. Itano, D. J. Wineland, and J. C. Bergquist, Science 319, 1808 (2008).

[4] C. W. Chou, D. B. Hume, J. C. J. Koelemeij, D. J. Wineland, and T. Rosenband, Phys. Rev. Lett. 104, 070802 (2010).

[5] C. W. Chou, D. B. Hume, M. J. Thorpe, D. J. Wineland, and T. Rosenband, Phys. Rev. Lett. 106, 160801 (2011).

[6] N. Hinkley, J. A. Sherman, N. B. Phillips, M. Schioppo, N. D. Lemke, K. Beloy, M. Pizzocaro, C. W. Oates, and A. D. Ludlow, Science 341, 1215 (2013).

[7] B. J. Bloom, T. L. Nicholson, J. R. Williams, S. L. Campbell, M. Bishof, X. Zhang, W. Zhang, S. L. Bromley, and J. Ye, Nature (London) 506, 71 (2014).

[8] I. Ushijima, M. Takamoto, M. Das, T. Ohkubo, and H. Katori, Nat. Photonics 9, 185 (2015).

[9] P. Gill, Phil. Trans. R. Soc. A 369, 4109 (2011).

[10] F. Riehle, arXiv:1501.02068.

[11] G. Santarelli, C. Audoin, A. Makdissi, P. Laurent, G. Dick, and A. Clairon, IEEE Trans. Ultrason. Ferroelectr. Freq. Control 45, 887 (1998).

[12] E. Peik, T. Schneider, and C. Tamm, J. Phys. B 39, 145 (2006).

[13] N. Herschbach, K. Pyka, J. Keller, and T. E. Mehlstäubler, Appl. Phys. B 107, 891 (2012).

[14] K. Pyka, N. Herschbach, J. Keller, and T. E. Mehlstäubler, Appl. Phys. B 114, 231 (2014).

[15] W. M. Itano, J. Res. Natl. Inst. Stand. Technol. 105, 829 (2000).

[16] H. Dehmelt, J. Phys. (Paris), Colloq. 42, C8-299 (1981).

[17] S. Schiller, Phys. Rev. Lett. 98, 180801 (2007).

[18] A. Derevianko, V. A. Dzuba, and V. V. Flambaum, Phys. Rev. Lett. 109, 180801 (2012).

[19] V. A. Dzuba, A. Derevianko, and V. V. Flambaum, Phys. Rev. A 86, 054502 (2012).

[20] V. A. Dzuba, V. V. Flambaum, and H. Katori, Phys. Rev. A 91, 022119 (2015).

[21] P. O. Schmidt, T. Rosenband, C. Langer, W. M. Itano, J. C. Bergquist, and D. J. Wineland, Science 309, 749 (2005).

[22] C. H. Bennett, H. J. Bernstein, S. Popescu, and B. Schumacher, Phys. Rev. A 53, 2046 (1996).

[23] P. Kaye and M. Mosca, J. Phys. A 34, 6939 (2001).

[24] K. Mølmer and A. Sørensen, Phys. Rev. Lett. 82, 1835 (1999).

[25] E. Solano, R. L. de Matos Filho, and N. Zagury, Phys. Rev. A 59, R2539 (1999).

[26] G. Milburn, S. Schneider, and D. James, Fortschr. Phys. 48, 801 (2000).

[27] C. F. Roos, New J. Phys. 10, 013002 (2008).

[28] K.-A. B. Soderberg and C. Monroe, Rep. Prog. Phys. 73, 036401 (2010).

[29] R. Blatt and C. F. Roos, Nat. Phys. 8, 277 (2012).

[30] T. R. Tan, J. P. Gaebler, Y. Lin, Y. Wan, R. Bowler, D. Leibfried, and D. J. Wineland, Nature (London) 528, 380 (2015).

[31] J. P. Home, in Advances in Atomic, Molecular, and Optical Physics edited by P. R. B. Ennio Arimondo and C. C. Lin (Academic Press, Boston, MA, 2013), Vol. 62, pp. 231-277.

[32] M. Wallquist, K. Hammerer, P. Rabl, M. Lukin, and P. Zoller, Phys. Scr. T137, 014001 (2009).

[33] G. Kurizki, P. Bertet, Y. Kubo, K. Mølmer, D. Petrosyan, P. Rabl, and J. Schmiedmayer, Proc. Natl. Acad. Sci. U.S.A. 112, 3866 (2015).
[34] For definiteness we illustrate our proposed scheme with Ramsey interrogation [cf. Fig. 2(a)], but note that arbitrary clock protocols involving entangled states and correlations between ion excitation probabilities can always be designed to yield $N_{C}+1$ measurement eigenvalues corresponding to the different possible numbers of excited ions [35]. A method to measure $\hat{N}$ can thus be used to read out any $N_{C}$-ion clock.

[35] V. Bužek, R. Derka, and S. Massar, Phys. Rev. Lett. 82, 2207 (1999).

[36] T. Rosenband, P. O. Schmidt, D. B. Hume, W. M. Itano, T. M. Fortier, J. E. Stalnaker, K. Kim, S. A. Diddams, J. C. J. Koelemeij, J. C. Bergquist, and D. J. Wineland, Phys. Rev. Lett. 98, 220801 (2007).

[37] G. J. Dick, in Proceedings of the 19th Annual Precise Time and Time Interval (PTTI) Application and Planning Meeting, 1987 (U.S. Naval Observatory, Washington, DC, 1988), pp. 133-147.

[38] G. J. Dick, J. D. Prestage, C. A. Greenhall, and L. Maleki, in Proceedings of the 22nd Annual Precise Time and Time Interval (PTTI) Applications and Planning Meeting, 1990 (U.S. Naval Observatory, Washington, DC, 1991), pp. 487508.

[39] See Supplemental Material at http://link.aps.org/supplemental/ 10.1103/PhysRevLett.116.013002, which includes Refs. [4046], for further details on transverse normal modes of multiion crystals, MS gates, algorithmic readout for arbitrary number of ions, and error estimates for algorithmic readout.

[40] D. Leibfried, B. DeMarco, V. Meyer, D. Lucas, M. Barrett, J. Britton, W. M. Itano, B. Jelenkovic, C. Langer, T. Rosenband, and D. J. Wineland, Nature (London) 422, 412 (2003).

[41] C. A. Sackett, D. Kielpinski, B. E. King, C. Langer, V. Meyer, C. J. Myatt, M. Rowe, Q. A. Turchette, W. M. Itano, D. J. Wineland, and C. Monroe, Nature (London) 404, 256 (2000).

[42] K. Kim, M.-S. Chang, R. Islam, S. Korenblit, L.-M. Duan, and C. Monroe, Phys. Rev. Lett. 103, 120502 (2009).

[43] G. Kirchmair, J. Benhelm, F. Zhringer, R. Gerritsma, C. F. Roos, and R. Blatt, New J. Phys. 11, 023002 (2009).

[44] T. Monz, P. Schindler, J. T. Barreiro, M. Chwalla, D. Nigg, W. A. Coish, M. Harlander, W. Hänsel, M. Hennrich, and R. Blatt, Phys. Rev. Lett. 106, 130506 (2011).

[45] M. A. Nielsen and I. L. Chuang, Quantum Computation and Quantum Information (Cambridge University Press, Cambridge, England, 2000).

[46] T. Choi, S. Debnath, T. A. Manning, C. Figgatt, Z.-X. Gong, L.-M. Duan, and C. Monroe, Phys. Rev. Lett. 112, 190502 (2014).

[47] Without loss of generality we can assume that $|N\rangle_{C}$ denotes the normalized, symmetric superpostion of $N$ clock ions in $|1\rangle$ and all others in $|0\rangle$; that is, $|N\rangle_{C}=$ $\left(\begin{array}{c}N \\ N_{C}\end{array}\right)^{-1 / 2} J_{+}^{N} \otimes_{i=1}^{N_{C}}|0\rangle$, where $J_{+}=\sum_{i=1}^{N_{C}} \sigma_{+}^{i}$.

[48] S.-L. Zhu, C. Monroe, and L.-M. Duan, Europhys. Lett. 73, 485 (2006).

[49] We assume $\Delta_{i j}^{\alpha \beta} \neq 0$, which is true for generic values of the detuning $\delta$.

[50] D. Kielpinski, B. E. King, C. J. Myatt, C. A. Sackett, Q. A. Turchette, W. M. Itano, C. Monroe, D. J. Wineland, and W. H. Zurek, Phys. Rev. A 61, 032310 (2000).

[51] G. Morigi and H. Walther, Eur. Phys. J. D 13, 261 (2001).

[52] D. McGloin, G. Spalding, H. Melville, W. Sibbett, and K. Dholakia, Opt. Express 11, 158 (2003). 